\title{
Design of reconfigurable doubly-curved canopy structure
}

\author{
F. Maden \& K. Korkmaz \\ Izmir Institute of Technology, Department of Architecture, Izmir, Turkey \\ Y. Akgün \\ Izmir Gediz University, Department of Interior Architecture, Izmir, Turkey
}

\begin{abstract}
In this paper, a new reconfigurable doubly-curved structure has been developed for a canopy roof. The proposed structure can transform itself to various configurations according to the activity and user requirements. It not only changes its shape from a planar geometry to doubly-curved geometries by means of actuators, but also becomes stable and carries loads. The main differences between proposed structure and similar deployable bar structures are that the proposed structure is more flexible with $2 D o F$ and it requires less number of bars and joints. To obtain the doubly-curved geometry, a novel method has been introduced. After discussing the kinematic behavior of the system, a set of structural analyses are performed in three different geometric configurations of the proposed structure.
\end{abstract}

\section{INTRODUCTION}

Movement has become a fact of life in twentieth century design culture. As a result of today's constantly changing activities, a need of adaptable space has emerged in architecture. To meet the changing functional, spatial or environmental needs, today's architecture seeks for adaptable structures ever than before. Based on the concept of adaptability, a series of kinetic structures which are capable of geometric transformations have been developed. Among the examples of these structures, the most impressive ones are deployable bar structures with single degree-offreedom $(D o F)$. These structures not only transform themselves from a compact configuration to a predetermined, expanded form, but also may become stable and carry loads (Pinero 1961; Escrig 1985; Gantes, 1989; Hoberman 1990; Kokawa \& Hokkaido 1997; Atake 2000; Van Mele et al. 2007; Akgün et al. 2010, 2011; Maden et al. 2011). Therefore, such structures may offer viable solutions for architectural applications, especially for temporary buildings, emergency shelters, exhibition halls, outdoor recreation facilities or sporting fields (Fig. 1).

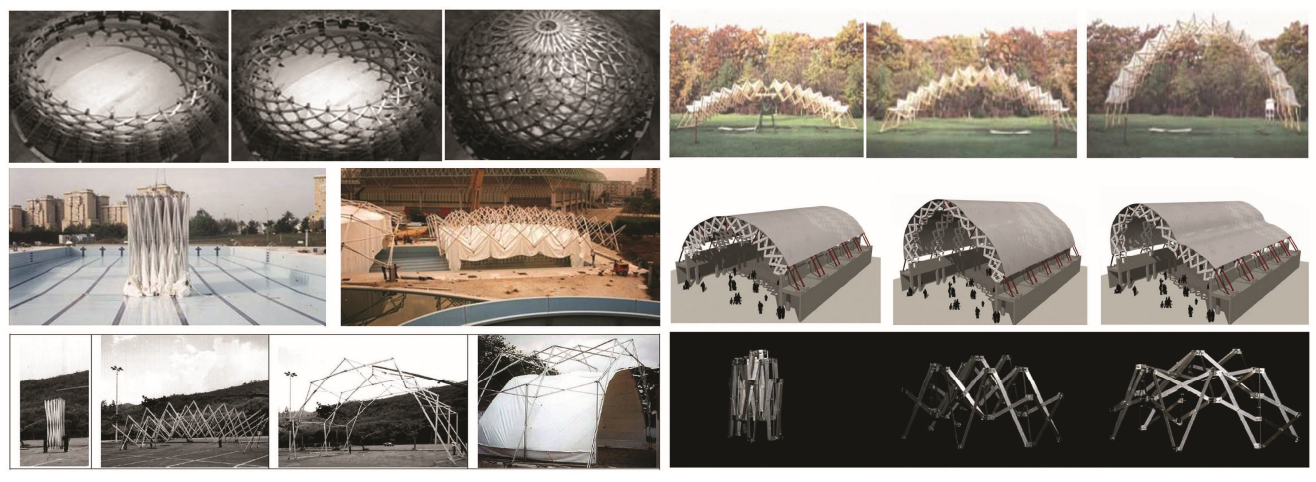

Figure 1. Examples of deployable bar structures 
Although many examples have been developed for deployable bar structures, only few have been constructed at full-scale for architectural applications. The main reason is that most of them are composed of scissor like elements (SLEs) which is a complex structural system to generate the desired surface. Moreover, present solutions are insufficient to constitute real form flexibility, because they are limited to certain geometric forms such as singly-curved vaults and doubly-curved synclastic domes. Due to the mathematical difficulty and mechanical complexity of their systems, doubly-curved anticlastic surfaces such as hyperboloid and hyperbolic paraboloid (HP) have been rarely used for deployable structures. In fact, anticlastic structures can be easily constructed by using simple straight bars rather than SLEs since their geometric forms can be generated by ruled surfaces (Fig. 2b, d).

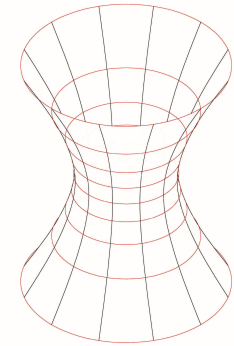

(a)

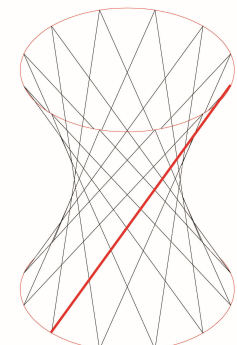

(b)

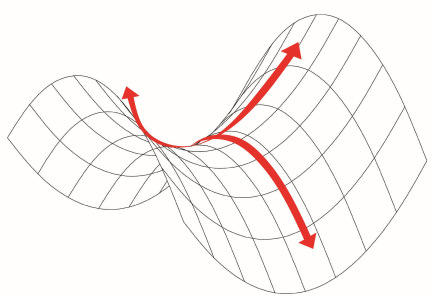

(c)

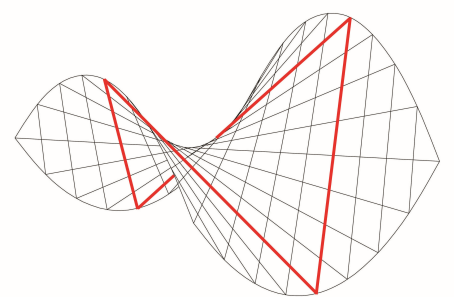

(d)

Figure 2. a) Hyperboloid generated by hyperbola; b) hyperboloid generated by straight line;

c) hyperbolic paraboloid generated by parabola; d) hyperbolic paraboloid generated by straight line

When compared to singly-curved or synclastic structures, it is seen that anticlastic structures are capable of resisting to various design loads through their curvatures and twists. Thus, their structural efficiency is more than the others. Considering the structural advantage of anticlastic structures and the ease of constructing their geometry by ruled surfaces, we have decided to use HP surface as a canopy roof structure. In this paper, we have introduced a new reconfigurable canopy structure which can change its shape according to the activity and user requirements. Because the HP is constructed by using ruled surface generation method, the system requires less number of bars and joints than the existing scissor structures. On the contrary to the current examples of single $D o F$ deployable bar structures, the proposed structure has $2 D o F$ which allows the system transform from planar geometry to various spatial ones. This type a transformation provides great flexibility for spaces.

\section{KINEMATIC ANALYSIS OF THE HP STRUCTURE}

The proposed HP structure is simply composed of straight bars and ring couplers (a sort of swivel coupler). The ring coupler consists of two rings which are connected to each other with a revolute joint. Each ring is connected to the bar with either a revolute joint $(R)$ or a cylindrical joint $(C)$. The kinematic diagram of the joints can be seen in Figure 3.

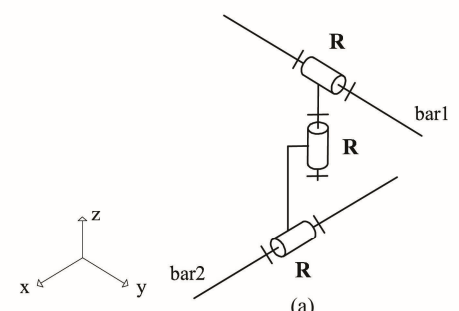

(a)

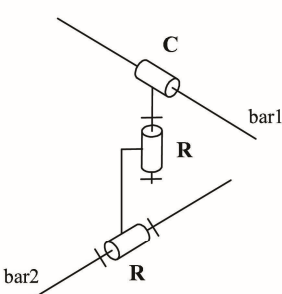

(b)

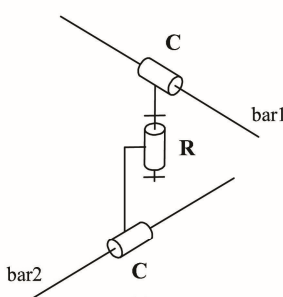

(c)

Figure 3. a) $3 R$ joint; b) $C 2 R$ joint; c) $2 C R$ joint 
The revolute joint between bar1 and bar2 allows a rotation around the z-axis. Its direction is perpendicular to the bar1 and bar2. If the joint on the bar is revolute, it only allows a rotation around the main axis of the bar. However, if it is a cylindrical joint, it allows both a rotation around the main axis of the bar and a translation over the bar.

To construct the $2 D o F$ reconfigurable HP structure, we have developed a novel method. At first, a frame structure composed of 4 bars and connected by $R$ joints has been generated (Fig. 4a).

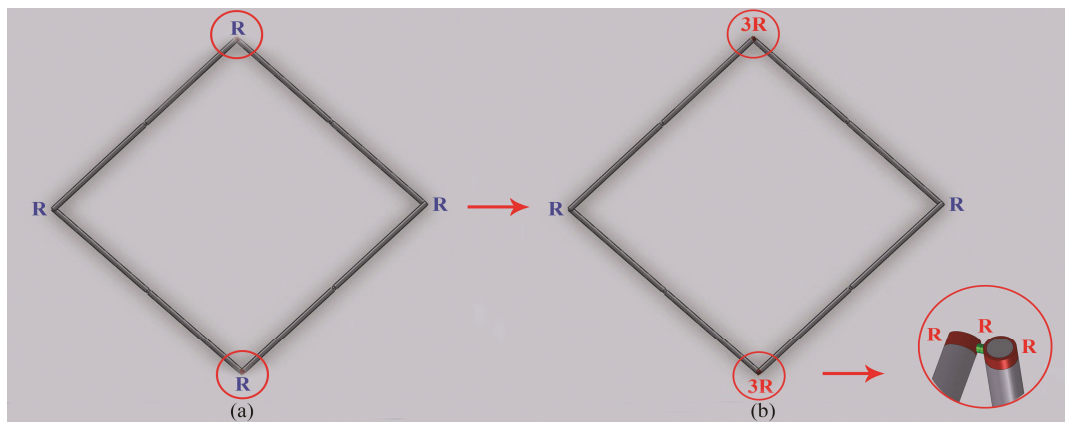

Figure 4. a) $4 R$ system; b) $R-3 R-R-3 R$ system

The mobility of the system has been calculated according to Alizade (2010) formula which is used for multi-loop mechanisms:

$$
M=\sum_{i=1}^{j} \mathrm{f}_{\mathrm{i}}-\sum_{k=1}^{L} \lambda_{k}+q-j_{p}
$$

where $j$ is the number of joints, $f_{i}$ is the relative joint motion, $L$ is the number of independent loops , $\lambda$ is the $D o F$ of space where the mechanism operates $(\lambda=2,3,4,5,6), q$ is the number of excessive elements and $j_{p}$ is the number of passive joints. To calculate $L$, Euler's formula has been used.

$$
L=j-l+1
$$

where $l$ is the number of links and $j$ is the number of joints. For the four bar system in Figure 4a, it is found that $\sum f_{i}=4, L=1, \lambda=3$ (the mechanism operates on plane). Therefore, it is calculated according to Eq. (1) that $M=4-(3 \times 1)+0-0=1$. This means that the system moves only on plane. To let it move in space, we have changed two of $R$ joints at opposite corner nodes with $3 R$ joints. The new system is composed of $R-3 R-R-3 R$ joints (Fig. $4 \mathrm{~b}$ ). According to Eq. (1), $\sum f_{i}=8$, $\lambda=6, L=1$ and $M=8-(6 \times 1)+0-0=2$. Because the system has $2 D o F$, it can move not only on plane, but also in space (Korkmaz et al. 2012). Three different deployed configurations of the structure can be seen in Figure 5.

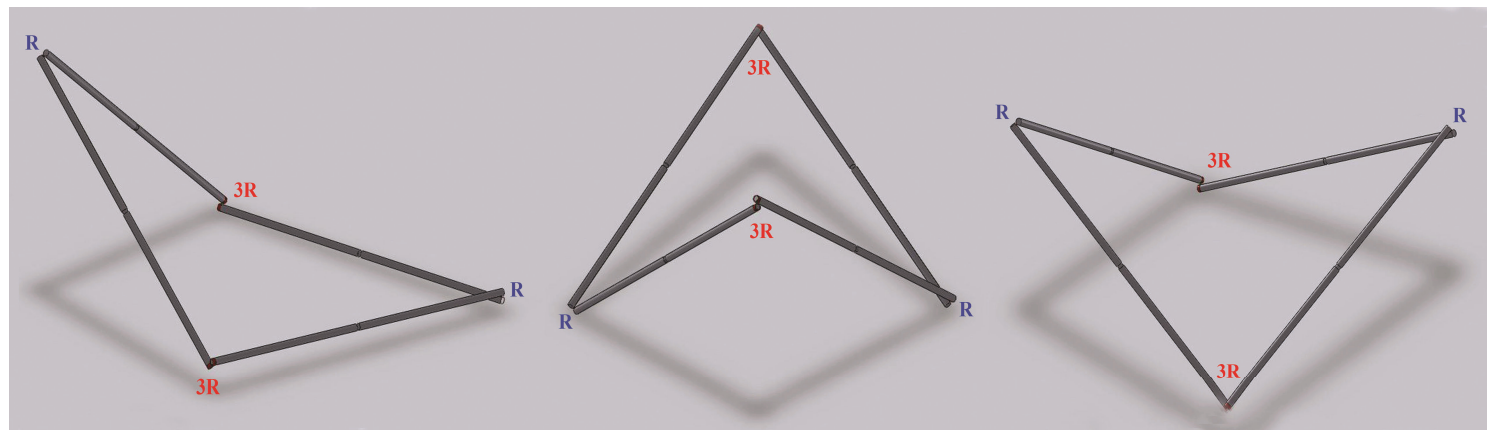

Figure 5. Three different deployed configurations of $2 D o F$ frame structure 
Second, a structural group has been added to the main frame structure whose mobility equals to zero. The structural group is composed of two bars which are connected to each other with a $R$ joint. There are 3 numbers of $C 2 R$ and a number of $2 C R$ joints at their end nodes (Fig. 6b). It is found that the required number of $C$ joint to generate $2 D o F 2 \times 2$-unit HP is 5 . Because the $C$ joint in the system defines the deployment behavior of the structure, it does not important at which end node $C$ joint is used. The only important thing is the number of $C$ joint in the system. For instance, if one of the $C$ joint in $2 C R$ joint is replaced with a $R$ joint, the $D o F$ of the system will automatically reduce to 1 . This makes the structure deployable rather than reconfigurable. Hence, it only deploys either on plane or in space. However, our aim is to create a reconfigurable structure which has various configurations. For this reason, we have used $5 C$ joints in the system. As it is seen in Figure 6c, the structural group has been connected to the main structure at the mid points of the edge bars. The system parameters are $\sum f_{i}=26, \lambda=6, L=4$. According to Eq. (1), the mobility of the system is calculated as $M=26-(6 \times 4)+0-0=2$. On the basis of this calculation, it can be said that adding a structural group to the main structure does not change the $D o F$ of the whole system. The planar system in Figure $6 \mathrm{c}$ can deploy to doubly-curved geometries. In Figure 7, three different deployed configurations of the 2DoF 2x2-unit HP is shown.

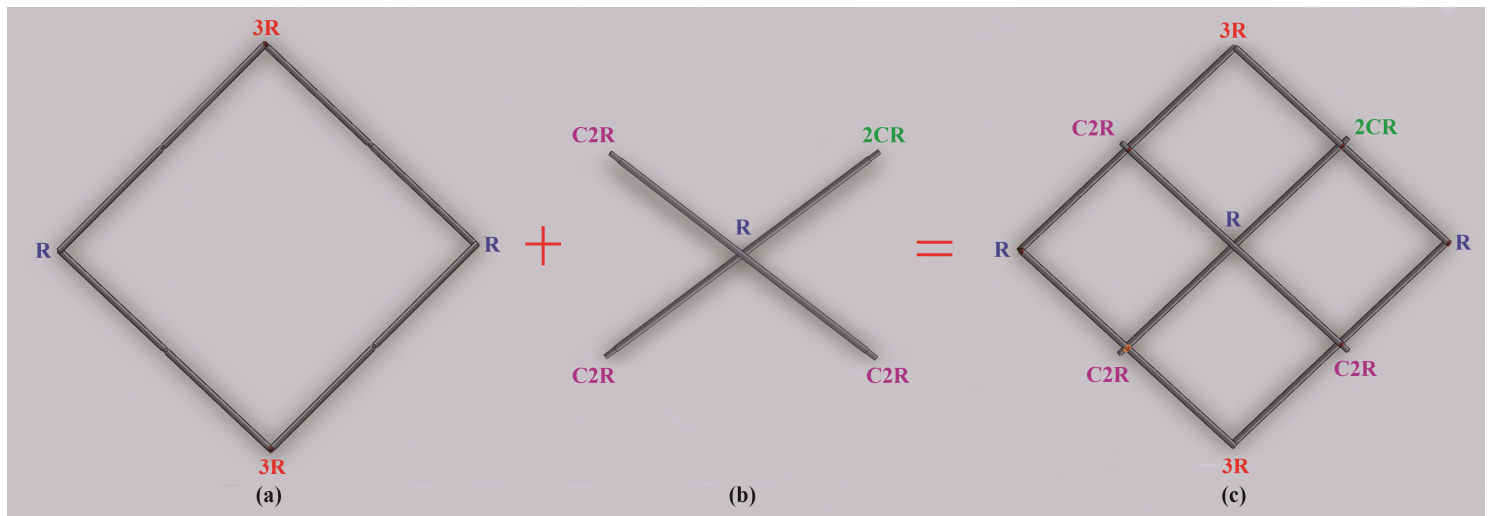

Figure 6. 2DoF 2x2-unit HP structure

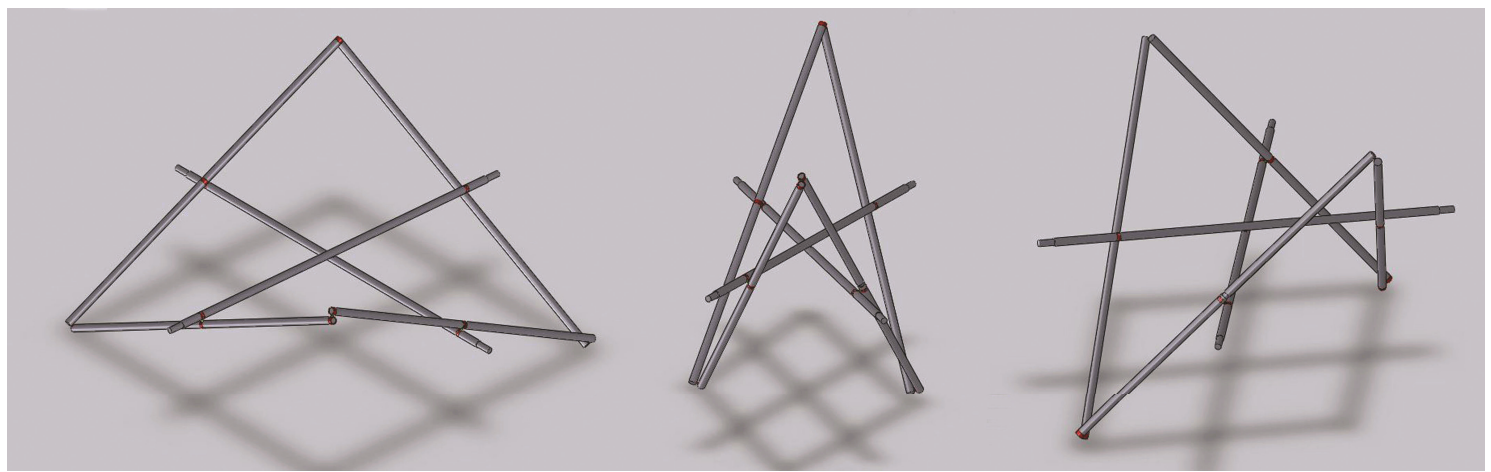

Figure 7. Three different deployed configurations of $2 D o F 2 \times 2$-unit HP structure

After proving that the structural group has no effect on the mobility of the system, we have developed some other models. For the next models, the bars in the structural groups have not been connected to each other at their mid points. The location of the connection point has been defined based on the division in the main structure. To obtain $3 \times 3$-unit HP structure, two structural groups have been added to the main structure as shown in Figure 8. However, this operation has introduced new intersections between the structural groups (Fig. 8c: node1 and node2). Although it is not necessary to connect them to each other, $2 C R$ joints have been used at these points to provide the system consistency. These two connections are excessive; therefore it is 
necessary to consider them as $q$ parameter in the formula when calculating the mobility of the system. With respect to Eq. (1), it is found that $\sum f_{i}=54, \lambda=6, L=9, q=2$ and $M=54-(6 \times 9)+2-0=2$. The $3 \times 3$-unit HP model in Figure 8c can deploy from planar geometry to different doublycurved geometries as in Figure 9.

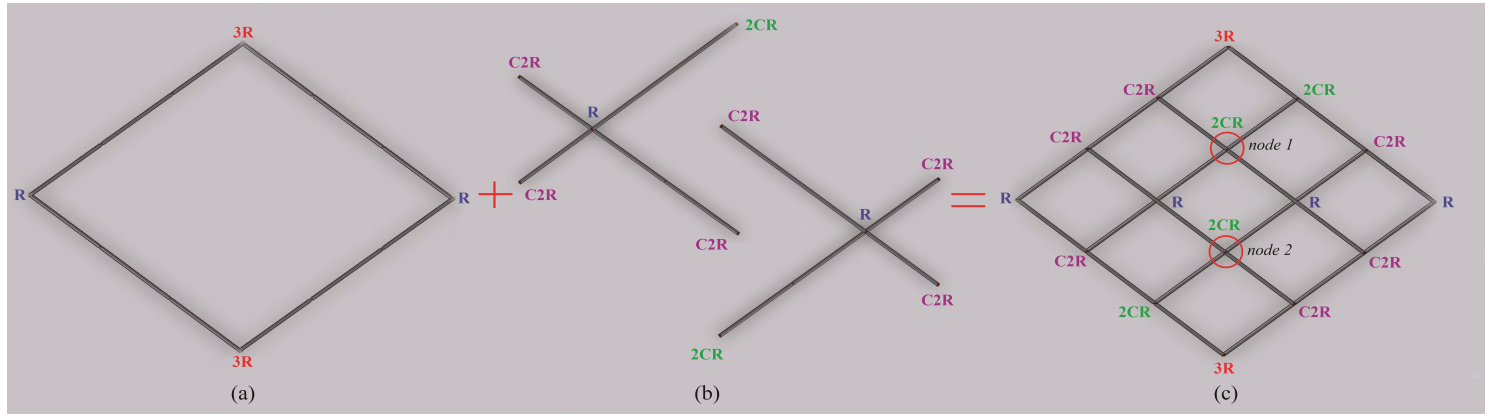

Figure 8. 2DoF 3x3-unit HP structure

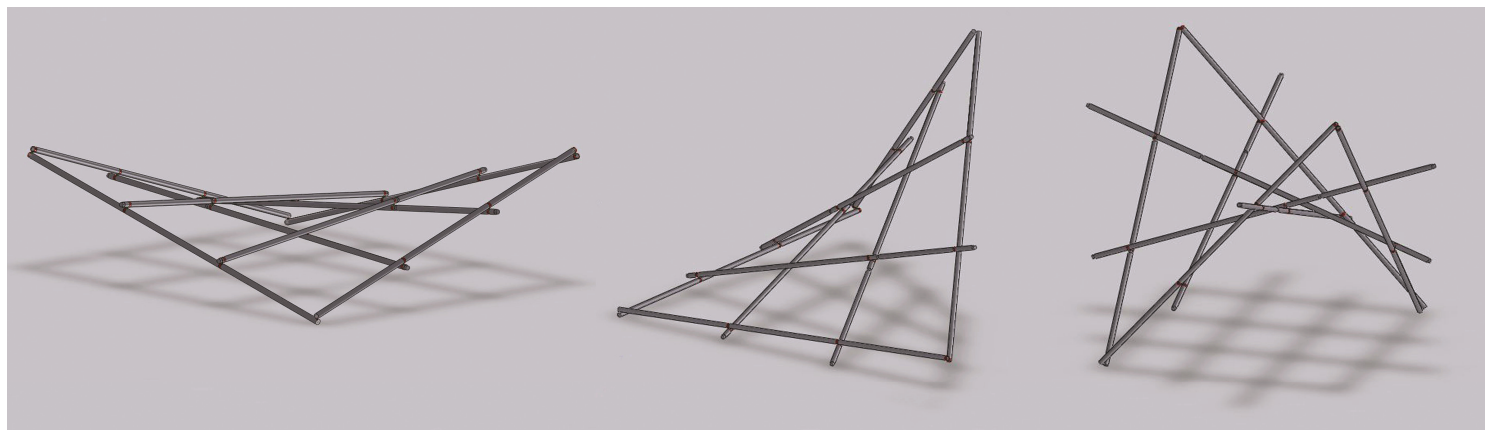

Figure 9. Three different deployed configurations of $2 D o F 3 \times 3$-unit HP structure

By following our surface generation method, we have increased the number of structural groups in the system for our next model. To obtain $4 \mathrm{x} 4$-unit HP, 3 structural groups have been used (Fig. 10b). For the intermediate intersections between the structural groups, $2 C R$ joints have been used as in 3x3-unit HP (Fig. 10c: nodes 1,2,3,4,5,6). There are 6 excessive connections in the system. According to Eq. (1), $\sum f_{i}=92, \lambda=6, L=16, q=6$. Therefore, $M=92-(6 \times 16)+6-0=2$. In Figure 11, three different deployed configurations of $2 D o F 4 \times 4$-unit HP structure can be seen. As a result, it can be claimed that any doubly-curved $2 D o F$ HP structure can be created by increasing the number of structural groups in the system.

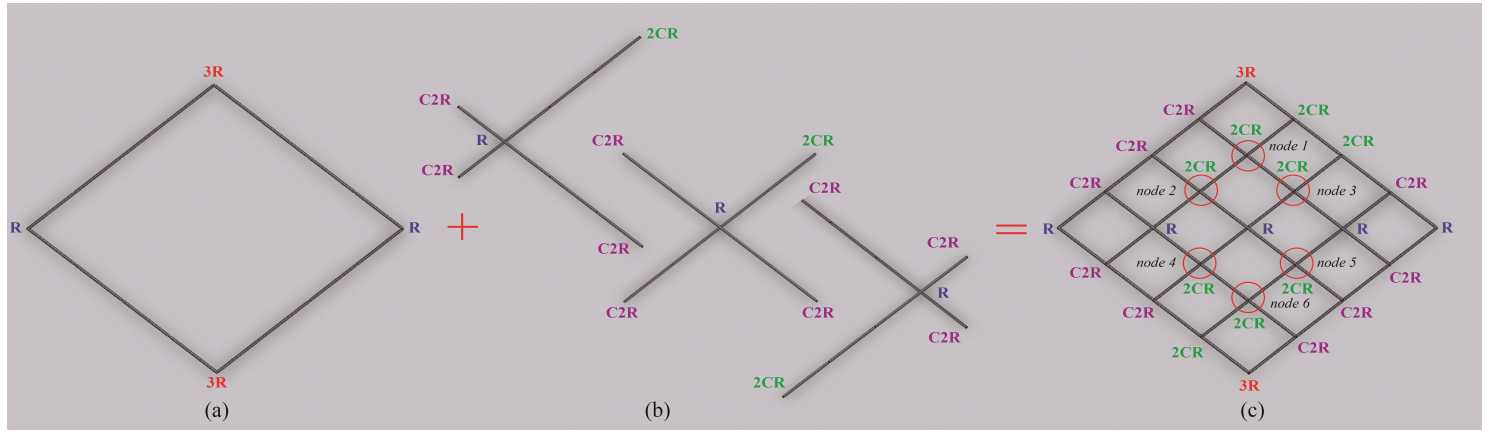

Figure 10. 2DoF 4x4-unit HP structure 


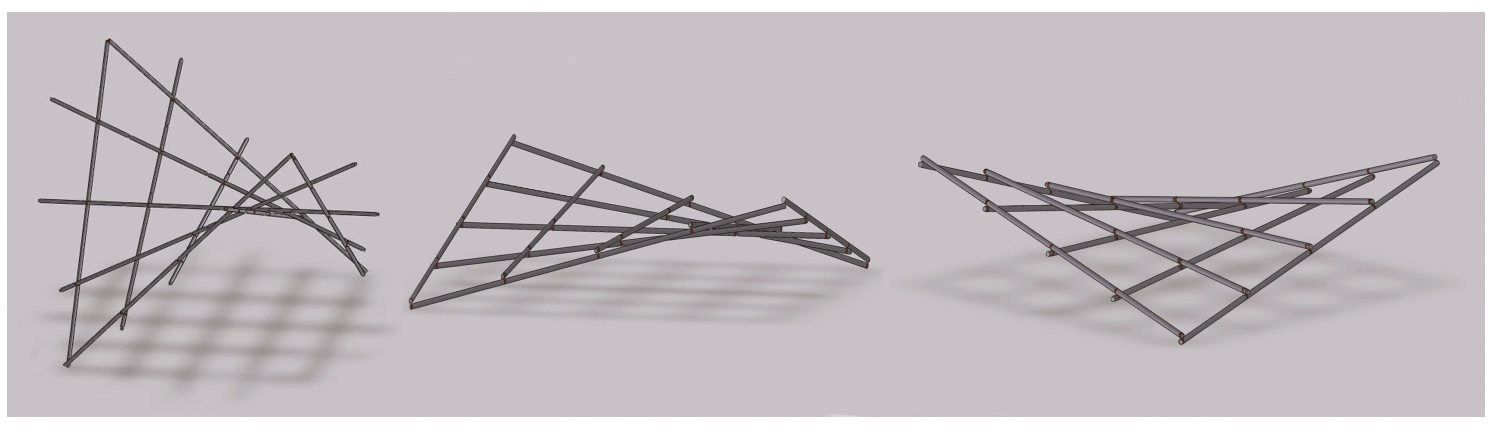

Figure 11. Three different deployed configurations of $2 D o F$ 4x4-unit HP structure

\section{PROPOSED STRUCTURE}

To construct the reconfigurable canopy roof structure, $2 D o F 4 \mathrm{x} 4$-unit HP structure has been selected. The proposed structure is composed of 10 identical tubular bars with a length of $10 \mathrm{~m}$, radius of $10 \mathrm{~cm}$ and thickness of $0.4 \mathrm{~cm}$. It has been connected to two columns at its two end nodes. The height of the columns is $5 \mathrm{~m}$. The span of the structure at planar configuration is $10 \mathrm{~m}$ in both directions which changes during the deployment process. The span ratio in the halfdeployed configuration is $1 / 2$ at a height of $5 \mathrm{~m}$. As a covering material of the structure, membrane has been used.

The movement of the structure is provided by means of two actuators which are like hydraulic piston. By controlling the actuators, the geometry of the structure can be adjusted according to the user requirement and desired space underneath. The structure is able to transform itself from a planar geometry to various doubly-curved geometries. When the system completes its transformation, it can be fixed at its supports in order to stabilize the structure. As a static system, it distributes the applied loads and self-weight of the structure. In Figure 12, four different configurations of the structure can be seen.
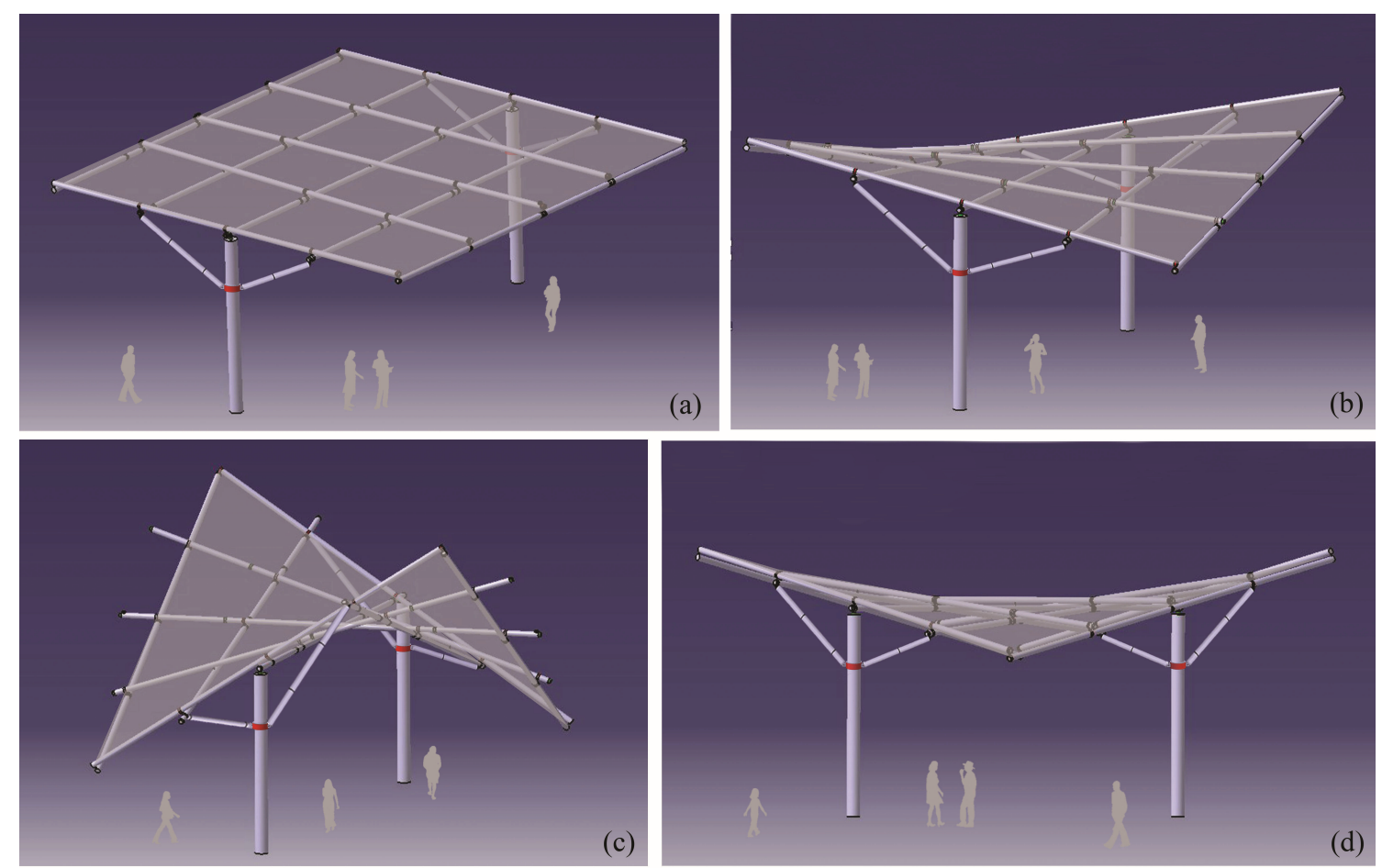

Figure 12. Four different deployed configurations of $2 D o F$ proposed structure 


\section{STRUCTURAL ANALYSIS OF THE PROPOSED HP STRUCTURE}

To understand the general structural behavior of the proposed HP structure under typical loading patterns, a set of structural analyses have been carried out. Three different geometric configurations of the HP have been determined for the analyses (Fig. 12a, b, c). Then, these configurations have been modeled in Oasys GSA software. Since the structure behaves like a mechanism, it develops no stresses during the deployment process. It is necessary to turn the mechanism into a structure in the deployed configurations. To block the movement of the system, the rotational $D o F \mathrm{~s}$ in the system has been removed by locking the actuators and fixing the distance between bracings. To guarantee the structural stability of the system, the nodes where the structure was connected to the columns and bracings have been fixed with pinned supports. By this means, the system acts as a structure and transfers loads.

In the analysis, S275 steel has been considered as the material of the bars whose elastic modulus equals to $210000 \mathrm{~N} / \mathrm{mm}^{2}$, Poisson's ratio is 0.3 and the yield stress is $275 \mathrm{~N} / \mathrm{mm}^{2}$. Based on Eurocode 1 guidelines, the loads have been determined. The response of the HP structure under vertical load such as self-weight of the structure and snow load and the horizontal load such as wind load have been simulated. For serviceability checks, a deflection limit of span/200 has been used which equals to $5 \mathrm{~cm}$ in our system.

The maximum stresses and displacements of the HP structure at three configurations are given in Table 1 and Table 2. According to the results, it is seen that the maximum vertical displacement is in the $1 \mathrm{st}$ configuration of the structure with $2.3 \mathrm{~cm}$. However, when the curvature starts to increase in both directions, the structure becomes more stable. The main reason is that the structure resists to loads through its curvatures and twists. Because the deflection limit is $5 \mathrm{~cm}$ in our system, the serviceability checks are satisfied for three configurations of the structure. Likewise, it is found that the maximum normal stress due to combined axial force and bending moment is $233 \mathrm{kN} / \mathrm{mm}^{2}$ in the 1 st configuration. Since the yield stress is $275 \mathrm{~N} / \mathrm{mm}^{2}$, the serviceability governs the design.

Table 1. Maximum displacements of the HP structure in 3 different configurations

\begin{tabular}{ll}
\hline Maximum displacements & Value $(\mathrm{cm})$ \\
\hline Configuration 1 & 2.3 \\
Configuration 2 & 2.1 \\
Configuration 3 & 1 \\
\hline
\end{tabular}

Table 2. Maximum stresses of the HP structure in 3 different configurations

\begin{tabular}{ll}
\hline Maximum displacements & Value $\left(\mathrm{kN} / \mathrm{mm}^{2}\right)$ \\
\hline Configuration 1 & 233 \\
Configuration 2 & 204 \\
Configuration 3 & 185 \\
\hline
\end{tabular}

\section{CONCLUSION}

This study has proposed a reconfigurable doubly-curved structure for a canopy roof which is ability to change its shape from planar geometry to various doubly-curved geometries. Rather than using the current systems based on SLEs, it has introduced a new novel method to generate the doubly-curved geometry by using the geometric design principles of ruled surfaces. By this means, it has provided to obtain the curved geometry with less number of bars and joints when compared to those current ones. On the contrary to single- $D o F$ deployable structures, it has developed a $2 D o F$ system which allows more flexibility. To obtain the $2 D o F$ system, kinematic analyses of different type of HP structures have been studied. Then, the structural properties of the proposed HP were analyzed. The analyses showed that the structural resistance of HP structure increases as the system deploys from planar geometry to doubly-curved geometries. 


\section{REFERENCES}

Akgün, Y., Gantes, C. J., Kalochairetis, K. E., \& Kiper, G. 2010. A novel concept of convertible roofs with high transformability consisting of planar scissor-hinge structures. Engineering Structures 32 (9): 2873-2883.

Akgün, Y., Gantes, C.J., Sobek, W., Korkmaz, K. \& Kalochairetis, K. 2011. A novel adaptive spatial scissor-hinge structural mechanism for convertible roofs. Engineering Structures 33 (4):1365-1376.

Alizade, R. 2010. Structural synthesis of robot manipulators. Proceedings of AZcIFToMM 2010, International Symposium of Mechanism and Machine Science. Izmir: Izmir Institute of Technology Press, pp.11-32.

Atake, K. 2000. New variations of the scissors technique. MARAS III: International Conference on Mobile and Rapidly Assembled Structures. Madrid: WIT Press, pp. 143-154.

Escrig, F. 1985. Expendable space structures. International Journal of Space Structures 1 (2): 79-91.

Eurocode 1: Actions on Structures. 2001. European Committee for Standardization (CEN).

Gantes, C., Connor, J.J., Logcher, R.D. \& Rosenfeld, Y. 1989. Structural analysis and design of deployable structures. Computers \& Structures 32 (3/4) : 661-669.

Hoberman, C. 1993. The iris dome. L'arca 7: 73.

Kokawa, T. \& Hokkaido, T. 1997. Cable scissors arch-marionettic structure: structural morphology, towards the new millennium. Proceedings of International Conference of IASS. Nottingham: University of Nottingham, pp.107-114.

Korkmaz, K., Akgün, Y. \& Maden, F. 2012. Design of a 2-DoF 8R linkage for transformable hypar structure. Mechanics Based Design of Structures and Machines 40 (1): 19-32.

Maden, F., Korkmaz K., Akgün Y. 2011. A Review of Planar Scissor Structural Mechanisms: Geometric Principles and Design Methods. Architectural Science Review 54: 246-257.

Pinero, E. 1961. Project for a mobile theatre. Architectural Design 12: 570.

Van Mele, T., De Temmerman, N., De Laet, L., \& Mollaert, M. 2007. Retractable roofs: scissor-hinged membrane structures. Proceedings of the TensiNet Symposium 2007 - Ephemeral Architecture: Time and Textiles. Milan: Liberia CLUP. 